\title{
Regenerative Electrode Interfaces for Neural Prostheses
}

\author{
Cort H. Thompson, BS, Marissa J. Zoratti, Nicholas B. Langhals, PhD, and Erin K. Purcell, PhD
}

Neural prostheses are electrode arrays implanted in the nervous system that record or stimulate electrical activity in neurons. Rapid growth in the use of neural prostheses in research and clinical applications has occurred in recent years, but instability and poor patency in the tissue-electrode interface undermines the longevity and performance of these devices. The application of tissue engineering strategies to the device interface is a promising approach to improve connectivity and communication between implanted electrodes and local neurons, and several research groups have developed new and innovative modifications to neural prostheses with the goal of seamless device-tissue integration. These approaches can be broadly categorized based on the strategy used to maintain and regenerate neurons at the device interface: (1) redesign of the prosthesis architecture to include finer-scale geometries and/or provide topographical cues to guide regenerating neural outgrowth, (2) incorporation of material coatings and bioactive molecules on the prosthesis to improve neuronal growth, viability, and adhesion, and (3) inclusion of cellular grafts to replenish the local neuron population or provide a target site for reinnervation (biohybrid devices). In addition to stabilizing the contact between neurons and electrodes, the potential to selectively interface specific subpopulations of neurons with individual electrode sites is a key advantage of regenerative interfaces. In this study, we review the development of regenerative interfaces for applications in both the peripheral and central nervous system. Current and future development of regenerative interfaces has the potential to improve the stability and selectivity of neural prostheses, improving the patency and resolution of information transfer between neurons and implanted electrodes.

\section{Introduction}

$\mathbf{N}$ EURAL PROSTHESES ARE devices implanted in the nervous system that are capable of stimulating or recording electrical activity from nearby neurons. Depending on the application, devices may interface with the peripheral or central nervous system (PNS or CNS). In the clinic, neural prostheses are used for a variety of treatment applications, including ameliorating the devastating deficits in motor function due to Parkinson's disease, intervening in seizure activity induced in intractable epilepsy, and returning sensory function to individuals who have lost vision or hearing. ${ }^{1-5}$ Investigative clinical applications include brainmachine interfaces, where the purpose is to restore motor and somatosensory function to victims of paralysis by directly controlling an exterior assistive device through signals recorded in the brain. ${ }^{6}$ Likewise, innovative designs are being explored to reconnect a patient's residual peripheral nerves with electrode arrays for direct control of upper- and lower-limb prostheses in amputees. ${ }^{7-10}$ Outside of the clinic, neural prostheses are invaluable research tools used to reveal the role of specific neurons and activation patterns in natural and pathological function. ${ }^{11-13}$ For all applications, stable, long-term communication between the device and the neuronal population of interest is desired.

While advanced neural interfaces continue to be developed, performance outcomes are variable. In the CNS, small signal amplitudes, signal instability, and signal loss over time are often observed. ${ }^{14,15}$ This may undermine the detection and decoding efficacy of brain-machine interfaces and closed-loop neurostimulation applications in which the stimulus is conditioned on a recorded neural signal.,15 There are a variety of potential reasons for signal loss and instability, including both biological and nonbiological origins, ${ }^{16,17}$ but the neural injury and inflammation that accompany device implantation are key contributing factors. Neuronal loss, signatures of neurodegeneration, and activation of neuronal cell death pathways have been observed in the tissue local to devices implanted in the CNS. ${ }^{18-20}$ Importantly, neuronal loss is most pronounced nearest the electrode surface, where a $\sim 40 \%$ decrease in neuronal density has been reported within the estimated recordable radius of the device following implantation in the brain. ${ }^{18,21}$ In the PNS, persistent inflammation, demyelination, Wallerian degeneration, neuroma

Department of Electrical and Computer Engineering, Neuroscience Program, Michigan State University, East Lansing, Michigan. 
formation, and encapsulation by connective tissue make maintaining patency in the electrode-tissue interface particularly challenging; axons have been observed to retract by over $1 \mathrm{~cm}$ from the wound site following penetrating electrode implantation. $^{22}$

To overcome these challenges, innovative bioengineering approaches to stabilize and/or regenerate the neuronal interface of implanted devices have been reported, where methods include the redesign of electrode array architecture and the incorporation of bioactive molecules, polymer coatings, cell-seeded scaffolds, or tissue constructs into the implant. Such strategies are amenable to the naturally regenerative environment of the PNS, but applications in the more challenging environment of the CNS have also exhibited success. In this study, we review the state of the art in approaches to regenerate and reconstruct the neuronelectrode interface, and we offer our perspective on the future growth in the development of regenerative neural prostheses.

\section{Peripheral Regenerative Nerve Interfaces}

Electrode interfaces in the PNS primarily include cuff electrodes, penetrating arrays, and regenerative approaches (for an overview of cuff electrodes and penetrating arrays, $\left.\mathrm{see}^{23}\right)$. As the cuff electrode wraps around the exterior of the nerve, it offers reasonable biocompatibility at the expense of selectivity in recording compound signals and stimulation of nerve fascicles. For penetrating arrays, the opposite is true: excellent integration with individual nerve fibers comes at the expense of a poorer tissue response due to the trauma and inflammation resulting from device implantation. Regenerative designs, which encourage axonal growth in close proximity to electrode sites, are a compromise alternative, offering improved biocompatibility while maintaining effective neuron-electrode contact. Various methods have been explored to encourage neural growth toward an electrode interface in the PNS, including the use of topographical cues, chemoattractants, material coatings, and biologics. These methods may be broadly categorized into three general schemes: (1) electrode architecture, (2) material coatings and incorporated biologics, and (3) biohybrid devices (literature summarized in Supplementary Fig. S1; Supplementary Data are available online at www.liebertpub.com/ teb).

\section{Electrode architecture}

While a primary aim of regenerative electrode interfaces is to enhance sensitivity of implanted electrodes by encouraging neuron-electrode proximity, selectivity in the communication between electrodes and the specific neuronal subtypes is a related goal of regenerative device design. In the PNS, bidirectional communication between segregated bundles of axons and individual electrode sites may enhance the resolution of sensory and motor information received and transmitted by the device. Roughly 40 years ago, the sieve electrode was conceived for the purpose of regenerating axons through a structure to make connections at electrode sites for communicating with neural tissue. This marked a substantial improvement in selectivity in comparison to the cuff electrode, which indiscriminately interfaced the entire nerve bundle. ${ }^{23}$ While initial designs were made of rigid materials such as silicon, ${ }^{24,25}$ Navarro and colleagues subsequently created sieve electrodes from flexible polyimide polymers. ${ }^{26,27}$ In an in vivo assessment of chronically implanted polyimide arrays, regenerating fibers were observed in all 30 implanted rats with little evidence of axonopathy, marking an improvement over silicon arrays. ${ }^{27}$ A natural spatial separation occurred between regenerating motor and sensory axons, where larger-diameter, slowergrowth motor axons tended to occupy the periphery of the device, and smaller, faster-regenerating sensory axons were found primarily in the center of the sieve. These results indicate some degree of separation of regenerating fiber subtypes, a feature that possibly could be leveraged for selective interfacing of efferents and afferents. Likewise, Castro et al.reported differential growth of certain axonal subtypes through sieve electrodes implanted in rat sciatic nerves: preferential growth of unmyelinated and sensory axons occurred in comparison to myelinated and motor axons. ${ }^{28}$ While the mechanisms for preferential axonal subtype growth were not immediately clear in their results, it may be that size exclusion plays a role, since growth of larger diameter and myelinated fibers may be impeded by small feature sizes. Revealing the potential mechanical, biological, and chemical factors responsible will inform the development of regenerative electrode interfaces with improved site-specific selectivity.

More recently, bidirectional interfaces have evolved into more precise instruments that allow regeneration of individual fascicles and axons through embedded microchannels. Lacour, Fawcett, and colleagues designed a microchannel electrode array (MCEA) featuring both polymeric microchannels for axonal regrowth and recording capability through integrated metal electrodes. ${ }^{29,30}$ The MCEA device is created by rolling a planar sheet of polyimide microchannels with individual $\sim 100 \times 100 \mu \mathrm{m}$ cross-sectional areas into a cylindrical tube structure. The MCEA device allowed for the regeneration of miniature nerve fascicles up to $5 \mathrm{~mm}$ long with their own vascular structures to grow through the implanted device. These fascicles within the MCEA maintained a similar structure and function to nerves regenerated through an ordinary silicone tube. An added benefit of this design is the spatial segregation of axonal bundles within the insulated microchannels; this feature may boost recorded signal by mitigating shunt loss through extracellular fluid ${ }^{31}$ and allow selective electrophysiological interfacing of particular subpopulations of axons (i.e., separation of afferent and efferent activity). Recordings from PNS neurons through a MCEA were later validated in rats in an acute setting. ${ }^{32}$ These studies established a proof of concept for the generation of more complex MCEAs incorporating regeneration-facilitating biologics.

A like-minded approach was explored by Bellamkonda and colleagues, who developed a grooved polydimethylsiloxane (PDMS)/SU-8 substrate incorporating stainless steel microwire electrodes for signal recording (Fig. 1A). ${ }^{33}$ Evaluation following implantation in the severed sciatic nerves of rats demonstrated several favorable outcomes: (1) scaffolds guided the growth of axons and supporting cells along the length of the embedded microchannels, (2) scaffolds stimulated the growth of essential Schwann cells and structural fibroblasts, potentially enhancing the integrity of regenerated neural tissue, (3) there was no evidence of neuroma formation, and (4) 


\section{A Micro-channel Electrode Array}

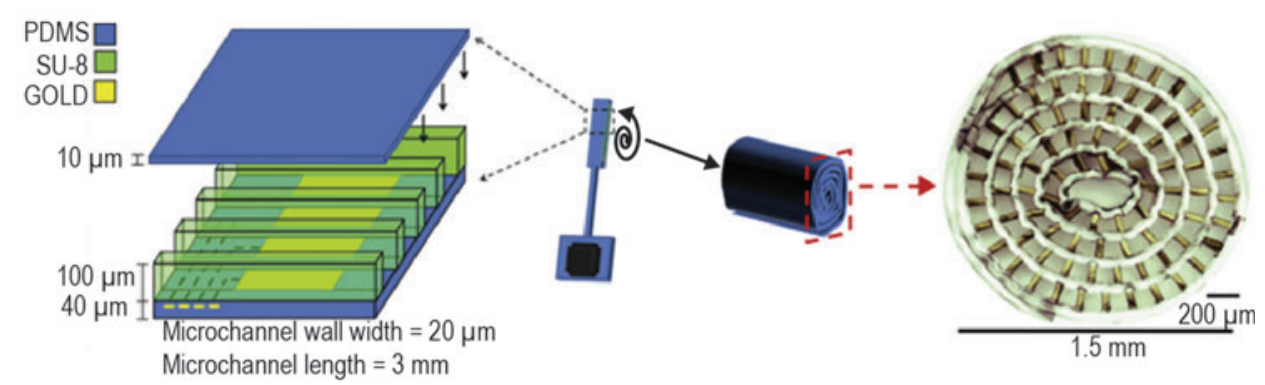

\section{B Bifurcating Arrays}
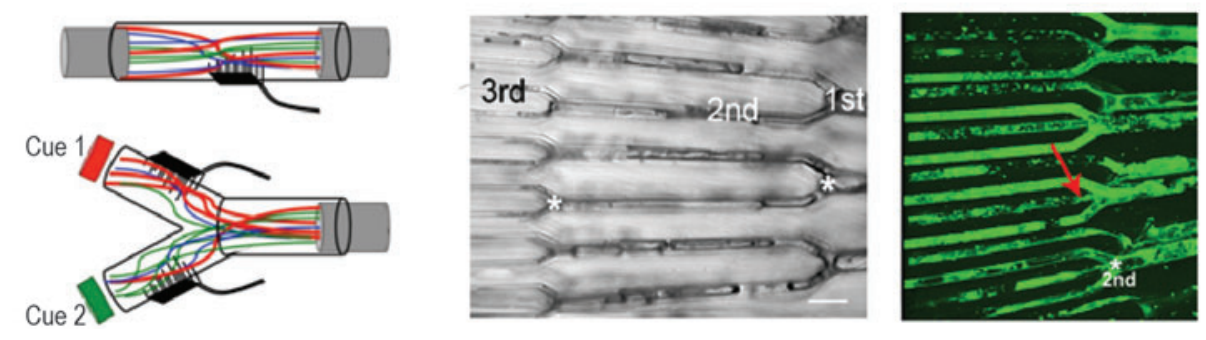

FIG. 1. Neural prosthesis architectures for improved integration with the peripheral nervous system. Microchannel-based devices guide regenerating axons and parcel outgrowth toward distinct target locations. (A) A polydimethylsiloxane (PDMS)/SU-8 microchannel array promotes axonal regeneration through microchannels and is capable of recording neuronal signals through embedded electrodes over a period of several months. (B) Left: Bifurcating microchannels can be combined with extrinsic cues at target locations to selectively interface distinct subtypes of neurons (indicated by green vs. red color). Middle and right: Multibranched and layered 3-D bifurcating arrays provide added complexity for separation of regenerating axons (labeled green in right panel) with bifurcation indicated by "*" and a red arrow indicating deeper channels within the array. Scale bar is $100 \mu \mathrm{m}$ in (B). Panel (A) is modified and reproduced with permission from Biomaterials $^{33}$ and IEEE/EMBS Proceedings. ${ }^{95}$ Panel (B) is modified and reproduced with permission from Frontiers in Neuroengineering ${ }^{34}$ (left panel) and the Journal of Neural Engineering ${ }^{35}$ (middle and right panels), (C) IOP Publishing. Reproduced with permission. All rights reserved. Color images available online at www.liebertpub.com/teb

electrical signals were recorded in vivo over a period of 5 months. ${ }^{33}$ This study concluded that the microchannel array design allowed for a considerable quantity of neural tissue regeneration with minimal scar tissue and inflammation, and future studies conducted over a longer time course (1 year) will be a useful extension to these results.

Expanding on the principles of intrafascicular peripheral interfaces, Romero-Ortega and colleagues have created Yshaped architectures that use an initially large opening in the microchannel followed by bifurcation into smaller channels (Fig. 1B, left). ${ }^{34}$ The central concept is that the device would parcel selected axonal fibers toward target sites, while allowing axons to regenerate in a more efficient and natural way. Furthermore, the device can be fabricated from a variety of materials (PDMS, agarose gels, polyurethane tubing) and can be modified to incorporate cues to entice fibers encoding specific sensory modalities to designated endpoints, improving the selectivity of the interface. ${ }^{34}$ In a similar approach, Stoyanova et al. crafted a 3D bifurcating electrode array from PDMS, where layers of bifurcating arrays were incorporated into the device architecture. ${ }^{35}$ When tested in vivo, these electrode arrays showed promise in facilitating bifurcation of large regenerated fascicles into smaller (almost singularly) separated axonal fibers (Fig. 1B, middle and right). These regenerated fibers contained both myelinated and unmyelinated fibers of varying diameter, which suggests the possible presence of motor, autonomic, and sensory neurites. These approaches show potential promise in guiding individual neurons to key electrode sites, presenting a novel approach to separating afferent and efferent contacts for bidirectional interfacing.

\section{Material coatings and incorporated biologics}

Polymer substrates with micron- and nanoscale topographical features can accelerate and guide axonal outgrowth to a desired target, ${ }^{36}$ and this phenomenon has been exploited to join electrodes and regenerating PNS axons. ${ }^{29,30,33-35,37}$ To provide the topographical cues to nearby neurons, some research groups have developed unique strategies using conductive polymers such as poly(3,4-ethylenedioxythiphene) (PEDOT) and poly(pyrrole) (PPy). ${ }^{38}$ In addition to reduced electrode site impedance, ${ }^{39}$ surfaces rendered from these materials can encourage neurite outgrowth from peripheral neurons when used to create scaffolds with neurite-promoting topographical cues. Abidian and coauthors reported enhanced neurite outgrowth of peripheral dorsal root ganglion (DRG) neurons on PEDOT and PPy nanotubes in comparison to conductive polymer film substrates. ${ }^{37}$ Electrical stimulation can further enhance results: stimulation increased neuritogenesis of rat pheochromocytoma (PC12) cells cultured on PPycoated poly(lactic-co-glycolic acid) nanofiber substrates in comparison to unstimulated cells. ${ }^{40}$ Additionally, studies have reported the development of conducting polymer/carbon 
nanotube (CNT) composites as electrode coatings, ${ }^{41}$ as these materials have exhibited favorable stability, biocompatibility, and conductivity characteristics. Lu et al. reported PC12 attachment and neurite outgrowth on PPy/CNT coatings in vitro, and coated electrodes were associated with increased neuronal density upon in vivo implantation in rats. ${ }^{42}$ More recently, PEDOT/CNT films were assessed for their utility in modifying a PNS interface, and it was found that coated electrodes exhibited improved neuronal responses at the interface and decreased expression of neuronal apoptosis markers following implantation into the DRG of rats. ${ }^{43}$ The mechanisms responsible for improved neuronal responses may be related to either active function (i.e., lower electrode impedance reducing injected power or the regenerative effects of electrical stimulation) or passive characteristics of the materials, including topographical, cellular adhesion, and cellular signaling cues; further study is required to identify the factors responsible. Interestingly, the degree of neurite outgrowth on the substrate may be affected by its passive conductivity ${ }^{44}$; identifying this or other potential mechanisms supporting neuronal growth would allow the ability to tune the regenerative response at the electrode interface based on the material substrate characteristics.

Incorporation of bioactive peptides in electrode material coatings is an additional avenue to regenerating and stabilizing the neural-electrode interface, where neurotrophic factors, anti-inflammatory drugs, and adhesive peptides have been explored for this purpose. ${ }^{45-50}$ Common means of introducing a biomolecule to an electrode material surface include surface adsorption, entrapment, or tethering through a functionalized group; for conductive polymers, inclusion as a dopant is an additional option. ${ }^{51}$ In the PNS, nerve growth factor (NGF) supports neuronal survival and neurite outgrowth, ${ }^{52}$ making it a promising candidate molecule to enhance regeneration at the electrode surface. NGF has been incorporated as a codopant in the electrochemical polymerization of PEDOT and PPy, where PC-12 cells responded with increased neurite outgrowth on the NGFcontaining polymer substrates. ${ }^{53}$ The use of NGF as a codopant in electrochemical polymerization has raised concerns regarding the long-term electrical and mechanical stability of the material, and some groups have pursued alternative approaches to delivering bioactive substances to the material coating. ${ }^{46}$ Gomez and Schmidt reported covalently linking NGF to an underlying PPy substrate, where enhancement of neurite outgrowth from attached PC-12 cells was similar with both NGF-immobilized PPy and soluble NGF. ${ }^{49}$ Green et al. entrapped NGF in PEDOT, in which laminin peptides were used as a codopant, presumably to leverage both the neurotrophic characteristics of NGF and the adhesive properties of laminin to improve the electrode-neuron interface. ${ }^{45}$ However, while bioactive NGF delivered from PEDOT doped with paratoluene sulfonate ( $\mathrm{p}-\mathrm{TS}$ ) promoted neurite outgrowth from PC12 cells, results with a laminin peptide-doped material were less encouraging, both in terms of the physical and biological assessment of the material. ${ }^{45}$ While the approach is promising, optimizing the long-term electrical, mechanical, and biological properties of bioactive conductive polymers remains an ongoing challenge. ${ }^{51}$

Richardson et al. have pursued a similar strategy to create regenerative electrode surfaces for cochlear implants, which are widely used to restore hearing in profoundly deaf patients through electrical stimulation of the auditory nerve. $^{47,48}$ In their approach, PPy coatings incorporate neurotrophin-3 (NT3), which is a neurotrophin known to support survival of auditory nerve cells (spiral ganglion neurons [SGNs]). ${ }^{54}$ An initial report demonstrated enhanced neurite outgrowth from SGNs cultured on PPy that incorporated NT3, and outcomes were further improved by applying electrical stimulation in a paradigm mimicking normal cochlear implant function. ${ }^{47} \mathrm{~A}$ subsequent assessment in vivo demonstrated increased SGN density and reduced thresholds of activation in guinea pigs receiving electrical stimulation from cochlear implants with PPy/pTS/NT3-coated electrodes in comparison to subjects receiving PPy/p-TS-coated devices. ${ }^{48}$ These results indicate a synergistic effect of neurotrophin release and electrical stimulation in promoting neuronal survival at the implanted electrode interface.

In addition to supporting the growth and maintenance of neurons near electrodes, spatially patterned delivery of neurotrophins may be used to selectively connect individual electrode sites with specific subtypes of neurons, providing improved selectivity to the neuron-electrode interface. In 2011, Lotfi et al. tested the utility of NGF and NT3 for the purpose of selective neuronal regeneration of nociceptive (NGF-responsive) and proprioceptive (NT3-responsive) fibers toward target locations (Fig. 1B, left). ${ }^{34}$ The ability to selectively grow individual neuronal subtypes along an electrode array may be a powerful tool to improve electrode array function and implantation, as certain electrode sites could be constructed to record or stimulate specific information (i.e., specific sensory modalities, or motor vs. sensory streams of information). The results presented indicated preferential neurite outgrowth of subtypes of neurons toward the expected target locations, providing a proof-of-concept support for this type of approach.

An alternative approach to incorporating biologics onto an electrode array is to incorporate new or existing supporting cell types to enhance neuronal regeneration and maintenance. Schwann cells are essential supporters of peripheral nerve tissue growth through secreting trophic factors and assisting regeneration following injury. ${ }^{55}$ While autologous Schwann cells may be difficult to harvest and isolate, mesenchymal stem cells are a promising alternative cell source for deriving Schwann-like cells and can be readily obtained from bone marrow or adipose tissue. ${ }^{56} \mathrm{Si}$ milarly to neurons, a variety of material substrate, chemical, and electrical cues can be used to further enhance the regeneration-promoting characteristics of supporting cells. For instance, Koppes et al. observed positive effects of electrically guided proximal Schwann cells on neurite outgrowth. ${ }^{57}$ Additionally, revascularization is another essential component of peripheral regeneration, and supporting vascular tissue regrowth has the potential to enhance electrode integration. Vascular endothelial growth factor (VEGF) is known to promote revascularization, axonal outgrowth, and Schwann cell proliferation. ${ }^{58}$ Utilizing Schwann cells cooperatively with VEGF may have the potential to enhance the electrode integration of target neural tissues by supplying supporting tissues permissive to neural regeneration. Furthermore, biomaterial scaffolds can be used to enhance the interaction of supporting cells and 
electrode interfaces. Behan and colleagues utilized singlewalled carbon nanotubes (SWCNTs) immersed within conductive hydrogels as a substrate for Schwann cell growth and observed responses in both 2D and 3D; they found that responses were particularly favorable when cells were embedded within the SWCNT hydrogel. ${ }^{59}$ Combining regenerative electrode interfaces with supporting cells potentially offers a more compatible and stable integration between electrodes and regenerated tissues.

\section{Biohybrid devices}

An alternative approach to regenerating the electrode interface involves the direct inclusion of living cells or tissue grafts onto the device (so-called biohybrid devices). ${ }^{60} \mathrm{~A}$ concept for a PNS biohybrid interface, which includes a polyimide sieve electrode array and a containment vessel for donor supporting cells, was conceptualized by Stieglitz et $a l .{ }^{60}$ An alternative approach to integrating biological material on a peripheral electrode interface has been pioneered by Cederna, Urbanchek, Langhals, and colleagues. ${ }^{61}$ In the regenerative peripheral nerve interface (RPNI), a segment of free muscle is grafted to the location of a transected nerve, and neurotized by the residual peripheral nerve (Fig. 2). ${ }^{61}$ The novelty of this design is that it addresses the biology of a severed peripheral nerve by providing it a new target to interface-the muscle graft. Since peripheral nerves continue to regenerate following an injury

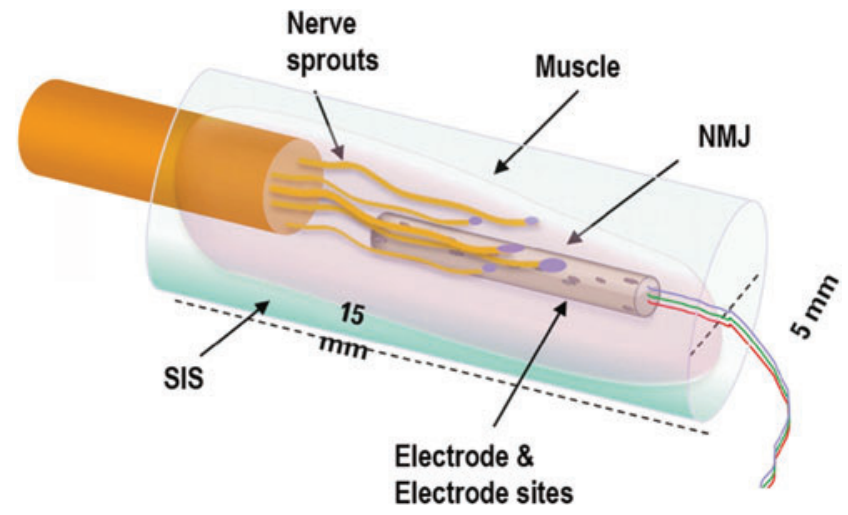

FIG. 2. Regenerative peripheral nerve interface (RPNI) to record prosthetic control signals from severed peripheral nerves. This biohybrid peripheral nerve interface is constructed by grafting small pieces of free muscle tissue to the end of divided or severed peripheral nerves. A small incision is placed within the muscle graft and the nerve is inserted. Electrodes are then placed on the surface or within the belly of the muscle to acquire prosthetic control signals and electrically stimulate afferent fibers for sensory feedback. The muscle serves as a biological target and protective substrate for the regenerating peripheral nerves and also functions as a signal amplifier by allowing larger electromyographic signals to be recorded from individual nerve fascicles compared to smaller electroneurographic signals typically acquired with surface or penetrating technologies. NMJ, neuromuscular junction; SIS, small intestine submucosal tissue. Above figure is reproduced with permission from Current Opinion in Otolaryngology \& Head and Neck Surgery. ${ }^{61}$ Color images available online at www .liebertpub.com/teb at $\sim 1 \mathrm{~mm}$ per day, grafting the muscle to the end of the nerve results in a minimal overall neural regeneration time. By grafting muscle to the end of the nerve, the standard signal pathway is disrupted, making this interface appropriate for amputees and distal nerve injuries. Translocation of the free muscle graft results in preliminary degeneration of the muscle graft while awaiting vessel inosculation and revascularization. In chronic studies in rats, the muscle is reinnervated by the peripheral nerve, and the formation of new neuromuscular junctions is evident. ${ }^{62}$ Compound muscle action potentials were successfully recorded from RPNIs over the entire 7-month evaluation period, with larger signals recorded from lower impedance, conducting polymer electrodes compared to standard stainless steel. ${ }^{62} \mathrm{~A}$ longer-term assessment over 14 months again demonstrated that RPNIs had positive histological outcomes and robust signal detection over the duration of the study. ${ }^{63}$ Further, unpublished data demonstrate biological viability of the interface exceeding 20 months in rodents. While RPNIs do not record signals as large as healthy muscles, due to the regeneration and reinnervation process, the muscle tissue serves as a biological amplifier by increasing the signal amplitude by 50-100 times compared to what can currently be recorded from state of the art peripheral nerve interfaces. Translation of this approach to humans is fairly straightforward. In the case of amputees, there are typically residual muscle pieces within the amputated limb that could be harvested for use in RPNI construction. If limited nearby tissue remains, muscle can be harvested from donor sites, such as the gluteus muscle. Given a small muscle harvest size of less than three centimeters, there is limited resultant donor site morbidity, which may regenerate from nearby myocytes.

\section{Central Regenerative Nerve Interfaces}

The CNS has little natural capacity to regenerate, and the insertion trauma, inflammation, and secondary injury response associated with chronically implanted microelectrode arrays present significant challenges to achieving effective long-term device function. Common sources of recording failures in the CNS include demyelination of the adjacent neurons, breakdown of the blood-brain barrier around the implant site, neuronal loss, degradation of the electrode, and formation of a glial sheath around implanted electrodes. ${ }^{64,65}$ Nonetheless, several approaches to regenerating and preserving neuronal function and density at the device interface have demonstrated success, where methods to modify electrodes include redesign of device architecture, use of material coatings, incorporation of biologics, and the development of biohybrid devices (literature summarized in Supplementary Fig. S2).

\section{Electrode architecture}

It is intuitive that smaller, less invasive device designs may produce a more favorable neuronal response to implanted CNS prostheses. These designs offer the dual benefit of reduced insertion damage due to a smaller footprint of the implanted device, as well as the capability to tap into neural circuitry with higher spatial resolution in a more distributed manner. In 2007, Seymour and Kipke reported increased neuronal density adjacent to finer-scale, lateral features of 
an open-architecture polymer neural probe implanted in the motor cortex of rats in comparison to tissue adjacent to the main shank (Fig. 3A). ${ }^{66}$ Further, implantation of functionalized devices demonstrated improved recording quality from electrode sites positioned on the lattice edge as compared to the faces and verified through computational modeling. ${ }^{67}$ This may be related to reduced non-neuronal density near the lateral features; reactive glia can produce inflammatory cytokines and chemokines with neurotoxic potential. ${ }^{18,68,69}$ In a similar approach, Skousen et al. found that neuronal loss was mitigated surrounding a lattice silicon neural probe featuring reduced surface area in comparison to a conventional, single shank silicon electrode implanted in the rat cerebral cortex (Fig. 3B). ${ }^{70}$ Next-generation devices fabricated from parylene-insulated carbon fibers with antibiofouling poly(ethylene glycol) methacrylate coatings have demonstrated robust recording quality and a minimal accompanying tissue response (Fig. 3C). ${ }^{71}$ While the smaller electrode sites necessitated by finer-scale geometries have the drawback of increased impedance, inclusion of conductive polymers mitigates this effect and allows improved signal detection. ${ }^{71,72}$ Somewhat paradoxically, it has been observed that the acute-phase production of inflammatory cytokines is increased surrounding smaller devices implanted in the rat brain; further work is needed to understand the relative contributions of gliosis, inflammation, and neuronal loss to recording quality to understand the potential impact of this finding. ${ }^{6}$ Nevertheless, smaller electrode sites facilitate high-density recording arrays with improved spatial sampling resolution, and there is ample room for further development of this approach.

\section{Material coatings and biologics}

Several groups have developed unique approaches to coat CNS electrode arrays with materials and biologics to improve device-tissue integration. He and Bellamkonda created a laminin-incorporating nanoscale coating through electrostatic layer-by-layer (LBL) fabrication; the coating is generated by alternating deposition of cation (polyethyleneimine) and anion (laminin) layers on an oxidecovered silicon substrate. ${ }^{73}$ The laminin-incorporating coating demonstrated superior adhesion and neurite outgrowth from cortical neurons cultured in vitro compared to alternative coatings and control wafers. A similar approach was developed by Wu et al., who reported successful culture of hippocampal and cortical neurons on hyaluronic acidbased films fabricated through LBL assembly. ${ }^{74}$ However, while some materials show promise in mitigating the tissue response in the in vitro setting, these impacts do not necessarily directly translate to findings observed in vivo. In a follow-up study, He and colleagues evaluated the nanoscale coating in vivo following implantation in rat brains, where it showed a positive impact on reducing gliosis at the 4 week time point, but no apparent effect on the neuronal density local to the implant. ${ }^{75}$ Likewise, parylene $\mathrm{C}$ is a biocompatible polymer commonly used to insulate recording electrodes, and it is has been shown to reduce attachment of microglia and macrophages to an underlying substrate in vitro, ${ }^{76}$ with the potential for results to be further improved by the inclusion of an antifouling coating. ${ }^{71}$ However, Winslow et al. compared parylene C-coated silicon

\section{A Open Architecture Probe}

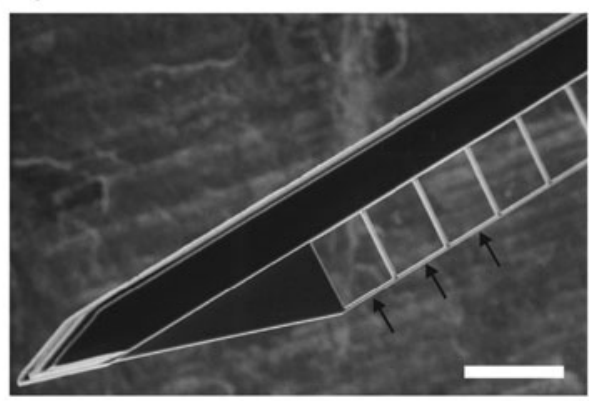

\section{B Lattice Probe}
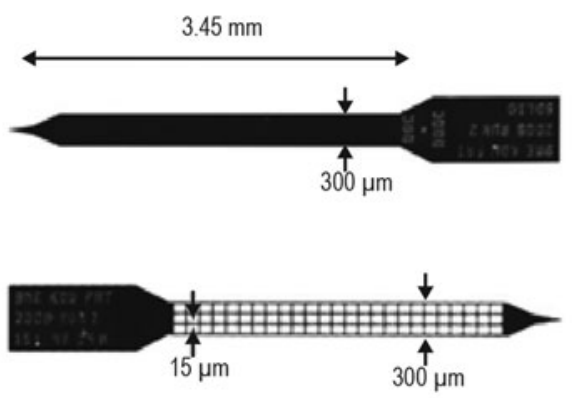

\section{Microthread Electrode}

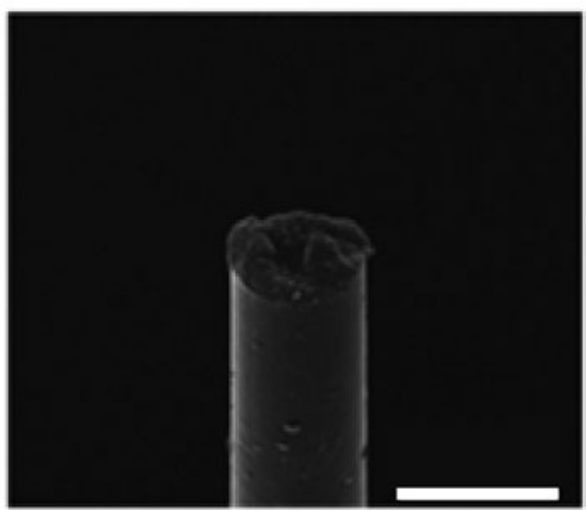

FIG. 3. Neural prosthesis designs incorporating finer-scale features for improved integration with the central nervous system (CNS). (A) An open-architecture neural probe includes support arms with dimensions measuring $4 \mu \mathrm{m}$ wide and $5 \mu \mathrm{m}$ thick as potential locations for electrode sites (arrows). The stiffer penetrating shank lateral to the support arms (appears black) facilitates surgical insertion. (B) In a similar strategy, the lattice probe presents reduced substrate surface area to local tissue. (C) An SEM image of a paryleneinsulated carbon fiber microthread electrode. Coating the electrode site with poly(3,4-ethylenedioxythiphene) (PEDOT) facilitates signal detection. The microscale geometries presented in (A-C) are each associated with an improved tissue response to the implant. Scale bars are $100 \mu \mathrm{m}$ in (A) and $10 \mu \mathrm{m}$ in (C). Panel (A) is reproduced with permission from Biomaterials. ${ }^{66}$ Panel (B) is modified and reproduced with permission from Progress in Brain Research. ${ }^{70}$ Panel (C) is reprinted by permission from Macmillan Publishers Ltd: Nature Materials, ${ }^{71}$ copyright (2012). 


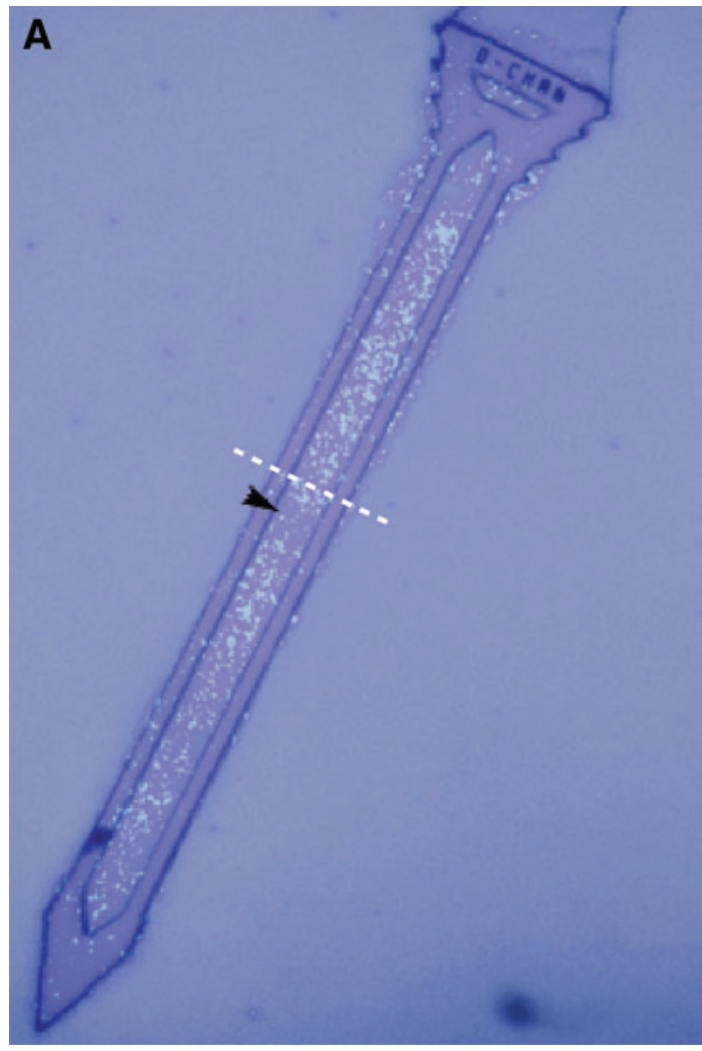

B
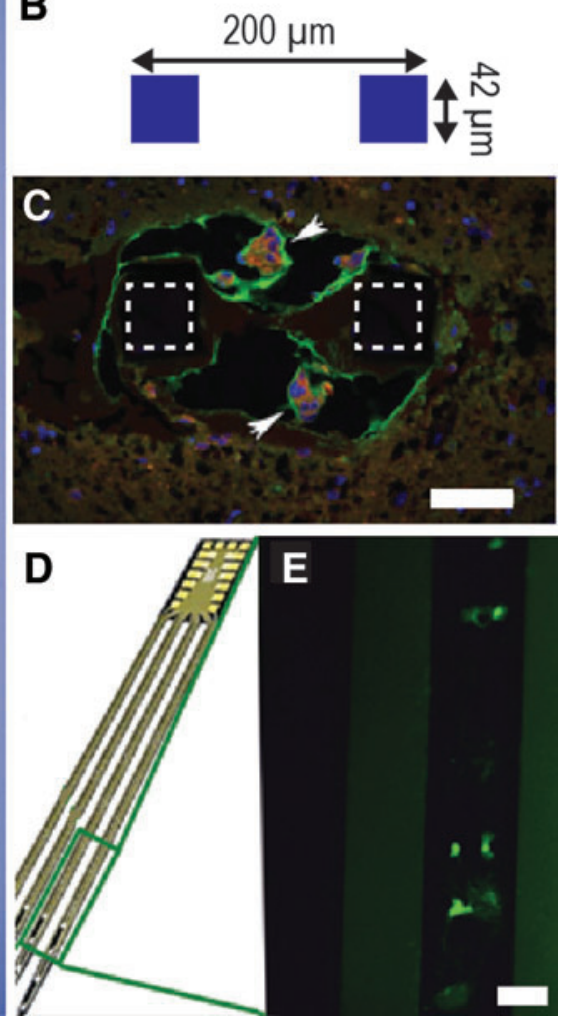

FIG. 4. Biohybrid neural prosthesis designs for the CNS. (A) Polymer probe houses a neural stem cell-seeded hydrogel scaffold (black arrowhead) along the shank of the device. Neural stem cells are counterstained with Hoechst (whitish-blue appearance). The dashed line indicates the cross-section illustrated in (B). (C) Immunohistochemistry showed evidence of graft cells (white arrowheads) within the device channel one day following implantation in a rat brain (red=doublecortin marker associated with neuronal precursors, green = marker for mouse-derived graft cells, blue $=$ Hoechst-stained nuclei). White dashed lines indicate location of device cross-section. (D, E) An alternative strategy for incorporating neural progenitors on neural prostheses immobilizes laminin on the surface of silicon arrays to facilitate cellular attachment to the device. Green fluorescent protein-expressing progenitors (E) are attached to the device area highlighted in (D). Scale bars are $50 \mu \mathrm{m}$ in $(\mathbf{C}, \mathbf{E})$. Panels $(\mathbf{A}-\mathbf{C})$ are modified and reproduced with permission from the Journal of Neural Engineering, ${ }^{87}$ (C) IOP Publishing. Reproduced with permission. All rights reserved. Panels (D-E)are modified and reproduced with permission from the Journal of Neurosurgery. ${ }^{88}$ Color images available online at www.liebertpub.com/teb

microelectrode arrays to uncoated arrays implanted in rat brains using a variety of quantitative immunohistochemical metrics for neuronal and non-neuronal outcomes over a 12week period, and there were no significant differences determined at any time point between the coated and uncoated electrodes. ${ }^{76}$ These results illustrate the challenges posed in translating in vitro effects to in vivo outcomes, where the intact brain poses an extraordinarily complex environment for effective regeneration at the device-tissue interface.

Recently, promising results have been reported in vivo with coatings created with the adhesion molecule, L1. L1 contributes many essential functions to the maintenance of the nervous system, where it is expressed in the majority of CNS neurons and has reported neuroprotective effects. ${ }^{77}$ It plays a role in specific cell-to-cell recognition, promotes the growth and development of complex connectivity in the nervous system, contributes to synaptic plasticity and CNS regenerative responses, and facilitates myelin formation. ${ }^{78}$ L1 has also been shown to promote neuronal growth and neurite extension, as well as reducing attachment of glial cells in vitro. ${ }^{79}$ Early in vivo results indicated that L1 may aid in decreasing mechanical failure and inflammation of electrodes implanted in the brain. ${ }^{80}$ Kolarcik et al. used this adhesion molecule to attempt to improve the tissueelectrode interface, where electrode tips were coated with parylene $\mathrm{C}$ before the immobilization of $\mathrm{L} 1$ onto the surface of the probe. ${ }^{81}$ In vitro, L1-modified electrodes decreased the inflammatory glial response and neuronal death associated with electrode implantation into the spinal cord and DRG of rats, ${ }^{81}$ and in vivo evaluation revealed similar results: "kill zones" characterized by reduced expression of neuronal markers surrounding electrodes were significantly decreased when L1-coated electrodes were used. ${ }^{81}$ Likewise, coating electrodes with L1 reduced gliosis and improved neuronal density surrounding devices implanted in rat motor cortex. ${ }^{77}$ Azemi et al. reported a pronounced improvement in neuronal density and neurofilament staining within the first $100 \mu \mathrm{m}$ of the probe surface over the entire 8 -week study period. ${ }^{77}$ Remarkably, while there was evidence of typical neuronal loss at the device interface surrounding control implants, no such reduction in neuronal density was evident surrounding L1-coated implants. ${ }^{77}$ 


\section{Biohybrid devices}

The neurotrophic cone electrode developed by Kennedy was a pioneering example of a biohybrid central neural prosthesis: a section of nerve housed within a glass electrode assembly was used to encourage neurite ingrowth near the gold recording wire. ${ }^{82}$ Following its initial description in 1989, several additional reports have validated the ability to obtain long-term recordings using cone electrode-based devices: signals were successfully recorded in rats and nonhuman primates, ${ }^{83,84}$ and recordings were obtained for several years in human subjects. ${ }^{85,86}$ More recently, concepts for neural stem and progenitor cell-seeded CNS prostheses have been reported, where incorporation on the device has been achieved through hydrogel scaffolding ${ }^{87}$ or attachment to $\operatorname{laminin}^{88}$ (Fig. 4). In the case of hydrogel scaffolding, an initial benefit to surrounding neuronal density was noted within the first week of transplantation, which may be related to release of neurotrophic factors by the engrafted cells. ${ }^{89}$ However, as is common in transplantation studies, ${ }^{90}$ detection of graft cells following implantation was limited to early time points, and survival of transplanted cells is likely to be a challenge with these approaches. However, these approaches offer a unique potential benefit, in that it may be possible to gain added control over the identity of specific subpopulations of neurons interfaced by individual electrode sites in addition to repopulating the device interface with new neurons (i.e., returning both enhanced sensitivity and selectivity to the interface). As technological advances for both cell grafting and unique device designs continue, it may be possible to create improved biohybrid devices, enabling both the repopulation and redefinition of the neuron-electrode interface.

\section{Future Prospects}

The rapid growth in the use of neural prostheses in the treatment of neurological diseases in the clinic, as well as their study in the research setting, attests to the incredible potential of these devices to reshape the future treatment and study of neurological diseases and injuries. Nonetheless, current designs suffer from poor integration between electrodes and neurons, where an extreme mismatch exists between the properties of the biological and synthetic substrates at the device interface. The use of regenerative approaches which promote or maintain neuronal growth and viability at the device interface are promising techniques for ensuring the long-term efficacy of neural prostheses. However, to fully realize the potential application of neural prostheses to solve problems in neurology and neuroscience, greater control and refinement over the neuron-electrode interface is desired. For instance, it would be extremely useful to achieve control over the interfaced neural subtypes, and regenerative interfaces could enable added capability and functionality in this context. For brain-machine interfaces, given the heterogeneity of individual neuronal subtypes in the brain, it may be useful to selectively connect and record from specific cell types with individual recording electrodes. In peripheral nerve interfaces for integrating with limb prostheses, it may be useful to separately connect motor and sensory fibers in a mixed nerve with distinct recording and stimulating electrodes, and even more useful to separately interface with individual sensory modalities, including proprioception, nociception, vibration, and touch. Furthermore, it may be the case that the neuronal population of interest is degenerated due to the underlying condition being treated (i.e., the cochlear implant stimulates degenerated auditory nerves $^{91}$ ); in this case, it may be possible to use regenerative approaches to repopulate the target neurons of stimulating devices. Finally, the tissue at the electrode interface demonstrates the re-emergence of markers associated with development and re-entry into the cell cycle, indicating plasticity in the local environment of neural prostheses. $^{87,92,93}$ Additionally, it has been shown that nanostructured materials can influence the synaptic transmission of attached neuronal networks, thus affecting the electrophysiological function of neurons at a synthetic interface. ${ }^{94}$ Leveraging native or induced plasticity in the environment local to implanted neural prostheses to gain additional control over device functionality is a relatively unexplored area that has the potential to further improve outcomes. With future growth in the development of new devices incorporating advanced materials, biologics, and device designs, it may be possible to gain unprecedented control over the longevity, resolution, and cell-type specificity of information transfer at the neuron-electrode interface.

\section{Acknowledgments}

The authors thank Dr. John Seymour for his assistance with preparing the open architecture probe figure. M.J.Z. received support from the Neuroscience Undergraduate Research Fellowship Program at MSU. This work was supported by the Department of Electrical and Computer Engineering at Michigan State University.

\section{Disclosure Statement}

No competing financial interests exist.

\section{References}

1. Rosin, B., Slovik, M., Mitelman, R., Rivlin-Etzion, M., Haber, S.N., Vaadia, E., et al. Closed-loop deep brain stimulation is superior in ameliorating parkinsonism. Neuron 72, 370, 2011.

2. Morrell, M.J. Responsive cortical stimulation for the treatment of medically intractable partial epilepsy. Neurology 77, 1295, 2011.

3. Zeng, F.G., Rebscher, S., Harrison, W., Sun, X., and Feng, H. Cochlear implants: system design, integration, and evaluation. IEEE Rev Biomed Eng 1, 115, 2008.

4. Weiland, J.D., and Humayun, M.S. Retinal prosthesis. IEEE Trans Biomed Eng 61, 1412, 2014.

5. Borton, D., Micera, S., Millán, J.R., and Courtine, G. Personalized neuroprosthetics. Sci Transl Med 5, 210rv2, 2013.

6. Hochberg, L.R., Bacher, D., Jarosiewicz, B., Masse, N.Y., Simeral, J.D., Vogel, J., et al. Reach and grasp by people with tetraplegia using a neurally controlled robotic arm. Nature 485, 372, 2012.

7. Rossini, P.M., Micera, S., Benvenuto, A., Carpaneto, J., Cavallo, G., Citi, L., et al. Double nerve intraneural interface implant on a human amputee for robotic hand control. Clin Neurophysiol 121, 777, 2010.

8. Raspopovic, S., Capogrosso, M., Petrini, F.M., Bonizzato, M., Rigosa, J., Di Pino, G., et al. Restoring natural sensory 
feedback in real-time bidirectional hand prostheses. Sci Transl Med 6, 222ra19, 2014.

9. Tan, D.W., Schiefer, M.A., Keith, M.W., Anderson, J.R., Tyler, J., and Tyler, D.J. A neural interface provides longterm stable natural touch perception. Sci Transl Med 6, 257ra138, 2014.

10. Clark, G.A., Wendelken, S., Page, D.M., Davis, T., Wark, H.A.C., Richard, A., et al. Using multiple high-count electrode arrays in human median and ulnar nerves to restore sensorimotor function after previous transradial amputation of the hand. In Engineering in Medicine and Biology Society (EMBC), 2014 36th Annual International Conference of the IEEE. 1977, 2014.

11. Jennings, J.H., and Stuber, G.D. Tools for resolving functional activity and connectivity within intact neural circuits. Curr Biol 24, R41, 2014.

12. Spira, M.E., and Hai, A. Multi-electrode array technologies for neuroscience and cardiology. Nat Nanotechnol 8, 83, 2013.

13. Obien, M.E.J., Deligkaris, K., Bullmann, T., Bakkum, D.J., and Frey, U. Revealing neuronal function through microelectrode array recordings. Front Neurosci 8, 2015.

14. Chestek, C.A., Gilja, V., Nuyujukian, P., Foster, J.D., Fan, J.M., Kaufman, M.T., et al. Long-term stability of neural prosthetic control signals from silicon cortical arrays in rhesus macaque motor cortex. J Neural Eng 8, 045005, 2011.

15. Perge, J.A., Homer, M.L., Malik, W.Q., Cash, S., Eskandar, E., Friehs, G., et al. Intra-day signal instabilities affect decoding performance in an intracortical neural interface system. J Neural Eng 10, 036004, 2013.

16. Prasad, A., Xue, Q.-S., Sankar, V., Nishida, T., Shaw, G., Streit, W.J., et al. Comprehensive characterization and failure modes of tungsten microwire arrays in chronic neural implants. J Neural Eng 9, 056015, 2012.

17. Prasad, A., Xue, Q.-S., Dieme, R., Sankar, V., Mayrand, R.C., Nishida, T., et al. Abiotic-biotic characterization of $\mathrm{Pt} / \mathrm{Ir}$ microelectrode arrays in chronic implants. Front Neuroeng 7, 2, 2014.

18. Biran, R., Martin, D.C., and Tresco, P.A. Neuronal cell loss accompanies the brain tissue response to chronically implanted silicon microelectrode arrays. Exp Neurol 195, 115, 2005.

19. Kozai, T.D.Y., Li, X., Bodily, L.M., Caparosa, E.M., Zenonos, G.A., Carlisle, D.L., et al. Effects of caspase-1 knockout on chronic neural recording quality and longevity: insight into cellular and molecular mechanisms of the reactive tissue response. Biomaterials 35, 9620, 2014.

20. McConnell, G.C., Rees, H.D., Levey, A.I., Gutekunst, C.A., Gross, R.E., and Bellamkonda, R.V. Implanted neural electrodes cause chronic, local inflammation that is correlated with local neurodegeneration. J Neural Eng 2009, 6, 056003.

21. Henze, D.A., Borhegyi, Z., Csicsvari, J., Mamiya, A., Harris, K.D., and Buzsáki, G. Intracellular features predicted by extracellular recordings in the hippocampus in vivo. J Neurophysiol 84, 390, 2000.

22. Christensen, M.B., Pearce, S.M., Ledbetter, N.M., Warren, D.J., Clark, G.A., and Tresco, P.A. The foreign body response to the Utah Slant Electrode Array in the cat sciatic nerve. Acta Biomater 10, 4650, 2014.

23. Del Valle, J., and Navarro, X. Interfaces with the peripheral nerve for the control of neuroprostheses. Int Rev Neurobiol 109, 63, 2013.
24. Mannard, A., Stein, R.B., and Charles, D. Regeneration electrode units: implants for recording from single peripheral nerve fibers in freely moving animals. Science 183, 547, 1974.

25. Akin, T., Najafi, K., Smoke, R.H., and Bradley, R.M. A micromachined silicon sieve electrode for nerve regeneration applications. IEEE Trans Biomed Eng 41, 305, 1994.

26. Lago, N., Udina, E., Ramachandran, A., and Navarro, X. Neurobiological assessment of regenerative electrodes for bidirectional interfacing injured peripheral nerves. IEEE Trans Biomed Eng 54, 1129, 2007.

27. Lago, N., Ceballos, D., Rodríguez, F.J., Stieglitz, T., and Navarro, X. Long term assessment of axonal regeneration through polyimide regenerative electrodes to interface the peripheral nerve. Biomaterials 26, 2021, 2005.

28. Castro, J., Negredo, P., and Avendaño, C. Fiber composition of the rat sciatic nerve and its modification during regeneration through a sieve electrode. Brain Res 1190, 65, 2008.

29. Lacour, S.P., Atta, R., FitzGerald, J.J., Blamire, M., Tarte, E., and Fawcett, J. Polyimide micro-channel arrays for peripheral nerve regenerative implants. Sens Actuat A Phys 147, 456, 2008.

30. Lacour, S.P., Fitzgerald, J.J., Lago, N., Tarte, E., McMahon, S., and Fawcett, J. Long micro-channel electrode arrays: a novel type of regenerative peripheral nerve interface. IEEE Trans Neural Syst Rehabil Eng 17, 454, 2009.

31. FitzGerald, J.J., Lacour, S.P., McMahon, S.B., and Fawcett, J.W. Microchannels as axonal amplifiers. IEEE Trans Biomed Eng 55, 1136, 2008.

32. Minev, I.R., Chew, D.J., Delivopoulos, E., Fawcett, J.W., and Lacour, S.P. High sensitivity recording of afferent nerve activity using ultra-compliant microchannel electrodes: an acute in vivo validation. J Neural Eng 9, 026005 , 2012.

33. Srinivasan, A., Tahilramani, M., Bentley, J.T., Gore, R.K., Millard, D.C., Mukhatyar, V.J., et al. Microchannel-based regenerative scaffold for chronic peripheral nerve interfacing in amputees. Biomaterials 41, 151, 2015.

34. Lotfi, P., Garde, K., Chouhan, A.K., Bengali, E., and Romero-Ortega, M.I. Modality-specific axonal regeneration: toward selective regenerative neural interfaces. Front Neuroeng 4, 1, 2011.

35. Stoyanova, I.I., van Wezel, R.J.A., and Rutten, W.L.C. In vivo testing of a 3D bifurcating microchannel scaffold inducing separation of regenerating axon bundles in peripheral nerves. J Neural Eng 10, 066018, 2013.

36. Purcell, E.K., Naim, Y., Yang, A., Leach, M.K., Velkey, J.M., Duncan, R.K., et al. Combining topographical and genetic cues to promote neuronal fate specification in stem cells. Biomacromolecules 13, 3427, 2012.

37. Abidian, M.R., Corey, J.M., Kipke, D.R., and Martin, D.C. Conducting-polymer nanotubes improve electrical properties, mechanical adhesion, neural attachment and neurite outgrowth of neural electrodes. Small 6, 421, 2010.

38. Green, R.A., Lovell, N.H., Wallace, G.G., and PooleWarren, L.A. Conducting polymers for neural interfaces: challenges in developing an effective long-term implant. Biomaterials 29, 3393, 2008.

39. Ludwig, K.A., Uram, J.D., Yang, J., Martin, D.C., and Kipke, D.R. Chronic neural recordings using silicon microelectrode arrays electrochemically deposited with a poly(3,4-ethylenedioxythiophene) (PEDOT) film. J Neural Eng 3, 59, 2006. 
40. Lee, J.Y., Bashur, C.A., Goldstein, A.S., and Schmidt, C.E. Polypyrrole-coated electrospun PLGA nanofibers for neural tissue applications. Biomaterials 30, 4325, 2009.

41. Keefer, E.W., Botterman, B.R., Romero, M.I., Rossi, A.F., and Gross, G.W. Carbon nanotube coating improves neuronal recordings. Nat Nanotechnol 3, 434, 2008.

42. Lu, Y., Li, T., Zhao, X., Li, M., Cao, Y., Yang, H., et al. Electrodeposited polypyrrole/carbon nanotubes composite films electrodes for neural interfaces. Biomaterials 31, 5169, 2010.

43. Kolarcik, C.L., Catt, K., Rost, E., Albrecht, I.N., Bourbeau, D., Du, Z., et al. Evaluation of poly(3,4-ethylenedioxythiophene)/carbon nanotube neural electrode coatings for stimulation in the dorsal root ganglion. J Neural Eng 12, 016008, 2015.

44. Malarkey, E.B., Fisher, K.A., Bekyarova, E., Liu, W., Haddon, R.C., and Parpura, V. Conductive single-walled carbon nanotube substrates modulate neuronal growth. Nano Lett 9, 264, 2009.

45. Green, R.A., Lovell, N.H., and Poole-Warren, L.A. Impact of co-incorporating laminin peptide dopants and neurotrophic growth factors on conducting polymer properties. Acta Biomater 6, 63, 2010.

46. Green, R.A., Lovell, N.H., and Poole-Warren, L.A. Cell attachment functionality of bioactive conducting polymers for neural interfaces. Biomaterials 30, 3637, 2009.

47. Richardson, R.T., Thompson, B., Moulton, S., Newbold, C., Lum, M.G., Cameron, A., et al. The effect of polypyrrole with incorporated neurotrophin-3 on the promotion of neurite outgrowth from auditory neurons. Biomaterials 28, 513, 2007

48. Richardson, R.T., Wise, A.K., Thompson, B.C., Flynn, B.O., Atkinson, P.J., Fretwell, N.J., et al. Polypyrrolecoated electrodes for the delivery of charge and neurotrophins to cochlear neurons. Biomaterials 30, 2614, 2009.

49. Gomez, N., and Schmidt, C.E. Nerve growth factorimmobilized polypyrrole: bioactive electrically conducting polymer for enhanced neurite extension. J Biomed Mater Res 81, 135, 2007.

50. Abidian, M.R., Kim, D.H., and Martin, D.C. Conductingpolymer nanotubes for controlled drug release. Adv Mater 18, 405, 2006.

51. Guimard, N.K., Gomez, N., and Schmidt, C.E. Conducting polymers in biomedical engineering. Prog Polym Sci 32, 876, 2007.

52. Chan, K.M., Gordon, T., Zochodne, D.W., and Power, H.A. Improving peripheral nerve regeneration: from molecular mechanisms to potential therapeutic targets. Exp Neurol 261, 826, 2014.

53. Kim, D.H., Richardson-Burns, S.M., Hendricks, J.L., Sequera, C., and Martin, D.C. Effect of immobilized nerve growth factor on conductive polymers: electrical properties and cellular response. Adv Funct Mater 17, 79, 2007.

54. Ernfors, P., Duan, M.L., ElShamy, W.M., and Canlon, B. Protection of auditory neurons from aminoglycoside toxicity by neurotrophin-3. Nat Med 2, 463, 1996.

55. Namgung, U. The role of Schwann cell-axon interaction in peripheral nerve regeneration. Cells Tissues Organs 200, 6, 2015.

56. Rodrigues, M.C.O., Rodrigues, A.A., Glover, L.E., Voltarelli, J., and Borlongan, C.V. Peripheral nerve repair with cultured schwann cells: getting closer to the clinics. ScientificWorldJournal 2012, 1, 2012.
57. Koppes, A.N., Nordberg, A.L., Paolillo, G.M., Goodsell, N.M., Darwish, H.A., Zhang, L., et al. Electrical stimulation of schwann cells promotes sustained increases in neurite outgrowth. Tissue Eng Part A 20, 494, 2014.

58. Sondell, M., Lundborg, G., and Kanje, M. Vascular endothelial growth factor has neurotrophic activity and stimulates axonal outgrowth, enhancing cell survival and Schwann cell proliferation in the peripheral nervous system. J Neurosci 19, 5731, 1999.

59. Behan, B.L., DeWitt, D.G., Bogdanowicz, D.R., Koppes, A.N., Bale, S.S., and Thompson, D.M. Single-walled carbon nanotubes alter Schwann cell behavior differentially within 2D and 3D environments. J Biomed Mater Res Part A 96, 46, 2011.

60. Stieglitz, T., Ruf, H., Gross, M., Schuettler, M., and Meyer, J.U. A biohybrid system to interface peripheral nerves after traumatic lesions: design of a high channel sieve electrode. Biosens Bioelectron 17, 685, 2002.

61. Langhals, N.B., Urbanchek, M.G., Ray, A., and Brenner, M.J. Update in facial nerve paralysis: tissue engineering and new technologies. Curr Opin Otolaryngol Head Neck Surg 22, 291, 2014.

62. Kung, T.A., Langhals, N.B., Martin, D.C., Johnson, P.J., Cederna, P.S., and Urbanchek, M.G. Regenerative peripheral nerve interface viability and signal transduction with an implanted electrode. Plast Reconstr Surg 133, 1380, 2014.

63. Langhals, N.B., Member, I., Woo, S.L., Moon, J.D., Larson J.V, Leach, M.K., et al. Electrically stimulated signals from a long-term regenerative peripheral nerve interface. In Engineering in Medicine and Biology Society (EMBC), 2014 36th Annual International Conference of the IEEE, 1989, 2014.

64. Polikov, V.S., Tresco, P.A., and Reichert, W.M. Response of brain tissue to chronically implanted neural electrodes. J Neurosci Methods 148, 1, 2005.

65. Kozai, T.D.Y., Jaquins-Gerstl, A.S. Vazquez, A.L., Michael, A.C., and Cui, X.T. Brain tissue responses to neural implants impact signal sensitivity and intervention strategies. ACS Chem Neurosci 6, 48, 2015.

66. Seymour, J.P., and Kipke, D.R. Neural probe design for reduced tissue encapsulation in CNS. Biomaterials 28, 3594, 2007.

67. Seymour, J.P., Langhals, N.B., Anderson, D.J., and Kipke, D.R. Novel multi-sided, microelectrode arrays for implantable neural applications. Biomed Microdevices 13, 441, 2011.

68. Karumbaiah, L., Saxena, T., Carlson, D., Patil, K., Patkar, R., Gaupp, E.A., et al. Relationship between intracortical electrode design and chronic recording function. Biomaterials 34, 8061, 2013.

69. Skousen, J.L., Bridge, M.J., and Tresco, P.A. A strategy to passively reduce neuroinflammation surrounding devices implanted chronically in brain tissue by manipulating device surface permeability. Biomaterials 36, 33, 2015.

70. Skousen, J.L., Merriam, S.M.E., Srivannavit, O., Perlin, G., Wise, K.D., and Tresco, P.A. Reducing surface area while maintaining implant penetrating profile lowers the brain foreign body response to chronically implanted planar silicon microelectrode arrays. Prog Brain Res 194, 167, 2011.

71. Kozai, T.D.Y., Langhals, N.B., Patel, P.R., Deng, X., Zhang, H., Smith, K.L., et al. Ultrasmall implantable composite microelectrodes with bioactive surfaces for chronic neural interfaces. Nat Mater 11, 1065, 2012. 
72. Ludwig, K.A., Langhals, N.B., Joseph, M.D., RichardsonBurns, S.M., Hendricks, J.L., and Kipke, D.R. Poly (3,4-ethylenedioxythiophene) (PEDOT) polymer coatings facilitate smaller neural recording electrodes. J Neural Eng 8, 014001, 2011.

73. He, W., and Bellamkonda R.V. Nanoscale neurointegrative coatings for neural implants. Biomaterials 26, 2983, 2005.

74. Wu, Z.-R., Ma, J., Liu, B.-F., Xu, Q.-Y., and Cui, F.-Z. Layer-by-layer assembly of polyelectrolyte films improving cytocompatibility to neural cells. J Biomed Mater Res Part A 81, 355, 2007.

75. He, W., McConnell, G.C., and Bellamkonda, R.V. Nanoscale laminin coating modulates cortical scarring response around implanted silicon microelectrode arrays. J Neural Eng 3, 316, 2006.

76. Winslow, B.D., Christensen, M.B., Yang, W.K., Solzbacher, F., and Tresco, P.A. A comparison of the tissue response to chronically implanted Parylene-C-coated and uncoated planar silicon microelectrode arrays in rat cortex. Biomaterials 31, 9163, 2010.

77. Azemi, E., Lagenaur, C.F., and Cui, X.T. The surface immobilization of the neural adhesion molecule L1 on neural probes and its effect on neuronal density and gliosis at the probe/tissue interface. Biomaterials 32, 681, 2011.

78. Hortsch, M. The L1 family of neural cell adhesion molecules: old proteins performing new tricks. Neuron 17, 587, 1996.

79. Azemi, E., Stauffer, W.R., Gostock, M.S., Lagenaur, C.F., and Cui, X.T. Surface immobilization of neural adhesion molecule L1 for improving the biocompatibility of chronic neural probes: in vitro characterization. Acta Biomater 4, 1208, 2008.

80. Zhong, Y., Yu, X., Gilbert, R., and Bellamkonda R V. Stabilizing electrode-host interfaces: a tissue engineering approach. J Rehabil Res Dev 38, 627, 2001.

81. Kolarcik, C.L., Bourbeau, D., Azemi, E., Rost, E., Zhang, L., Lagenaur, C.F., et al. In vivo effects of L1 coating on inflammation and neuronal health at the electrode-tissue interface in rat spinal cord and dorsal root ganglion. Acta Biomater 8, 3561, 2012.

82. Kennedy, P.R. The cone electrode: a long-term electrode that records from neurites grown onto its recording surface. J Neurosci Methods 29, 181, 1989.

83. Kennedy, P.R., Mirra, S.S., and Bakay, R.A.E. The cone electrode: ultrastructural studies following long-term recording in rat and monkey cortex. Neurosci Lett 142, 89, 1992.

84. Kennedy, P.R., and Bakay, R.A. Activity of single action potentials in monkey motor cortex during long-term task learning. Brain Res 760, 251, 1997.

85. Bartels, J., Andreasen, D., Ehirim, P., Mao, H., Seibert, S., Wright, E.J., et al. Neurotrophic electrode: method of as- sembly and implantation into human motor speech cortex. J Neurosci Methods 174, 168, 2008.

86. Kennedy, P. Reliable neural interface: the first quarter century of the neurotrophic electrode. Conf Proc IEEE Eng Med Biol Soc 2012, 3332, 2012.

87. Purcell, E.K., Seymour, J.P., Yandamuri, S., Kipke, D.R. In vivo evaluation of a neural stem cell-seeded prosthesis. J Neural Eng 6, 026005, 2009.

88. Azemi, E., Gobbel, G.T., and Cui, X.T. Seeding neural progenitor cells on silicon-based neural probes. J Neurosurg 113, 673, 2010.

89. Purcell, E.K., Singh, A., and Kipke, D.R. Alginate composition effects on a neural stem cell-seeded scaffold. Tissue Eng Part C 15, 541, 2009.

90. Dimmeler, S., Ding, S., Rando, T.A., and Trounson, A. Translational strategies and challenges in regenerative medicine. Nat Med 20, 814, 2014.

91. Khan, A.M., Whiten, D.M., Nadol, J.B., and Eddington, D.K. Histopathology of human cochlear implants: correlation of psychophysical and anatomical measures. Hear Res 205, 83, 2005.

92. Purcell, E.K., Thompson, D.E., Ludwig, K.A., and Kipke, D.R. Flavopiridol reduces the impedance of neural prostheses in vivo without affecting recording quality. J Neurosci Methods 183, 149, 2009.

93. Kozai, T.D.Y., Gugel, Z., Li, X., Gilgunn, P.J., Khilwani, R., Ozdoganlar, O.B., et al. Chronic tissue response to carboxymethyl cellulose based dissolvable insertion needle for ultra-small neural probes. Biomaterials 35, 9255, 2014.

94. Cellot, G., Toma, F.M., Kasap Varley, Z., Laishram, J., Villari, A., Quintana, M., et al. Carbon nanotube scaffolds tune synaptic strength in cultured neural circuits: novel frontiers in nanomaterial-tissue interactions. J Neurosci 31, 12945, 2011.

95. Srinivasan, A., Liang, G., and Bellamkonda, R.V. "Regenerative microchannel electrode array for peripheral nerve interfacing." Neural Engineering (NER), 2011 5th International IEEE/EMBS Conference on. IEEE, 2011.

Address correspondence to: Erin K. Purcell, PhD

Department of Electrical and Computer Engineering Neuroscience Program Michigan State University 428 S. Shaw Lane Room 2120 East Lansing, MI 48824

E-mail: epurcell@msu.edu

Received: June 16, 2015

Accepted: September 14, 2015

Online Publication Date: November 23, 2015 